\title{
Study of droplet characteristics and spray deposition during coating produced by an electrostatic rotary bell sprayer on OpenFoam
}

\author{
Mohammad-Reza Pendar ${ }^{1, *}$, José Carlos Páscoa ${ }^{2}$ \\ ${ }^{1,2}$ Electromechanical Engineering, University of Beira Interior, Covilhã, Portugal \\ * m.reza.pendar@ubi.pt
}

\begin{abstract}
The current work seeks to investigate the unsteady spraying flow characteristics around the high-speed electrostatic rotary bell sprayer (ERBS). The description information is about the distribution, size, velocity, charge-tomass ratio and disintegration process of the injected paint droplets. Also, the present study contains a systematic analysis of the toroidal vortex pattern, recirculation length (RL), trajectories of the droplet and the overspray phenomenon. The droplet electrostatic spraying process and fluid dynamics are simulated by implementing a comprehensive EulerianLagrangian model and Navier-Stokes equations, respectively. The current efficient algorithm accurately computed the interaction between the shaping airflow dynamic, electrostatic field and lagrangian paint droplet in the spraying process under the framework of the OpenFOAM package. Unsteady flow is calculated by using a large eddy simulation (LES) turbulence model. This study precisely focuses on the formation of the paint film distribution on the inner surface of the bell cup and workpiece. The effect of different breakup models on the distribution and size of the droplets during the coating is reported. Also, a function for the wall film is implemented to capture the thickness of the impinging particles on the body surface. The droplet disintegrations pattern and airflow distribution and velocity near the bell cup region compared with the experimental results and a suitable agreement were observed.
\end{abstract}

Keywords: Electrostatic rotary bell sprayer (ERBS); OpenFOAM; Large eddy simulation (LES); Droplet breakup

\section{INTRODUCTION}

During these years the electrostatic rotary bell sprayers because of their high-quality and uniform paintings, proper spatial atomization characteristics, higher transfer efficiency (TE), a considerable reduction in the material costs, decrease the impacts on the environment and improvement of the performance are broadly used in the coating industries. Because of the extensive use of the ERBSs in the automotive industry and the existence of many open questions about their operational mechanism, it is needed to be studied for reaching a deeper understanding. Several studies investigated the droplets transport process during the spraying and formed film thickness $[1,2]$. A few other investigations were conducted about the procedure of the electrical space charge [3], wall film approaches [4], droplet size distributions [5] and shaping airflow effect [6] for the electrostatic rotary bell sprayers. Some authors have been studied the charged particle acceleration effect due to the presence of an electric field $[7,8]$. Pendar and Páscoa $[9,10]$ simulated the electrostatic spray transfer processes with an Eulerian-Lagrangian approach in the OpenFOAM package. Their investigation predicts the flowfield characteristics and particle distribution accurately and also compared with the available experimental results. Darwish Ahmad et al. [11] visualized a novel Schlieren base of shaping air (SA) flow around the ERBS, without injection of the paint droplets. Hence, their experimental study optimized the SA for improving the quality of the spray. Also, they [5] reported experimental measurements as a database for evaluating the fundamentals of physical treatment in the ERBS by changing a broad range of operational parameters. Stevenin et al. [6] experimentally investigated the rotation frequency and its effect on the aerodynamics around the ERBS. They found that by a significant increase in the rotational speed a regime change has happened which increases the length of the recirculation zone. Wilson et al. [12] considered the breakup mechanisms by high-speed imaging near the edge of the ERBS in a wide range of rotational speed. They indicated that the higher rotational speed reduced the length of the ligament and Sauter mean diameters (SMD) around $26.8 \%$ and $22.3 \%$, respectively. Fujimoto et al. [13] shows that the droplet diameter which is predicted by the breakup approaches strongly affected the spray shape. The flowfield over a highspeed rotary propulsion device is also studied by Rami and Pascoa $[14,15]$. These authors mentioned the credibility of this turbulence model to fairly predict the existing turbulent structures. Comparing different turbulence models for flow phenomenon on a highly curved blade surface shows that the LES turbulence model admitted the higher accuracy in the numerical results [16-18]. LES approach is more appropriate to 
capture the details of the computed flow and demonstrate the principle of the internal flow [19-21].

The current paper basically deals with a significant number of parameters in different operational conditions in which the electrostatic spray flow depends on that. Furthermore, the effect of different breakup model especially in the region near to the rotary cup surface where the droplets distributed from there. The latter result is implementing a wall film function in the OpenFOAM solver for estimating the thickness of the impinging droplets on the target surface.

\section{GOVERNING EQUATIONS}

The numerical algorithm which is implemented in the current study generally can be divided into three different phases: Continuous phase (compressible Navier- Stokes equations, LES turbulence model), discrete phase (Lagrangian approach) and Electrostatic field (Poisson's equation). In the current work compressible Navier-Stokes equations is used for a continuous phase. Continuity and momentum equations for the airflow are given as bellow:

$$
\begin{aligned}
& \partial_{t} \rho+\nabla \cdot(\rho u)=0 \\
& \rho \partial_{t} u+\rho((\mathrm{u} . \nabla) \mathrm{u})=-\nabla P+\nabla \cdot\left[\mu\left(\nabla \mathrm{u}+(\nabla \mathrm{u})^{T}\right)+\nabla\left(-\frac{2 \mu}{3} \nabla \cdot \mathrm{u}\right)+\right. \\
& F_{s t}+F_{e s}+S .
\end{aligned}
$$

In the current simulation, the external electric field is produced by the potential difference between the bell-cup and the grounded workpiece. A Poisson's equation modeled the electric field around the bell, which is described by relation among the electric field intensity (E) and space-charge density $\left(\rho^{q}\right)$ :

$$
\varepsilon^{\prime} \nabla . E=\rho^{q}
$$

Where $\varepsilon^{\prime}$ is the electrical permittivity of the gas phase. The electric field intensity representing in terms of potential $(\phi)$ is governed as follow:

$$
\nabla^{2} \phi=-\frac{\rho^{q}}{\varepsilon^{\prime}}, \vec{E}=-\nabla \phi .
$$

The charge on each individual particles is:

$$
Q_{i}=m_{P_{i}} \rho_{\mathrm{m}_{\mathrm{i}}}^{\mathrm{q}}=\left(\rho_{P} V_{P_{\mathrm{i}}}\right) \rho_{\mathrm{m}_{\mathrm{i}}^{\mathrm{q}}}^{\mathrm{q}}
$$

Where $\rho_{P}, V_{P_{\mathrm{i}}}$ and $\rho_{\mathrm{m}_{\mathrm{i}}}^{\mathrm{q}}$ are density, volume and charge-to-mass ratio of an individual particle, respectively. Finally, the electric force per each unit mass is defined as:

$$
F_{E}=q_{P} E ., E . \nabla \rho^{q}=-\frac{\rho^{q^{2}}}{\varepsilon^{\prime}}
$$

The charged droplet motion is described by solving differential equations for calculating of the droplet velocity, size and position at each instant. The equation for the motion of the sprayed droplets is formulate as follow:

$$
\begin{aligned}
& m_{P_{i}} \frac{\partial \vec{u}_{P_{i}}}{\partial t}=\frac{3}{4} C_{d} \frac{\rho_{f}}{\rho_{P_{i}}} \frac{m_{P_{i}}}{2 r}\left|\left(\vec{u}_{f}-\vec{u}_{P_{i}}\right)\right|\left(\vec{u}_{f}-\vec{u}_{P_{i}}\right)+ \\
& \left(\rho_{P_{i}}-\rho_{f}\right) V_{P_{i}} \vec{g}+\frac{\rho_{f} V_{P_{i}}}{2} \frac{\partial\left(\vec{u}_{f}-\vec{u}_{p}\right)}{\partial t}+\vec{E} q_{P_{i}}
\end{aligned}
$$

Different part in the right side of the formula are stokes drag, buoyancy, electric force and added mass force, respectively. Where $\rho_{f}$ are the, $\mathrm{r}$ and $\mathrm{q}_{\mathrm{p}}$ are the fluid density, radius and the charge of the particles, respectively. $C_{D}$ is the drag coefficient that depends on the droplet's Reynolds number determined as:

$$
C_{D}=\left\{\begin{array}{lc}
\frac{24}{\operatorname{Re}_{P}}, & \text { if } \operatorname{Re}_{P}<1 \\
\frac{24}{\operatorname{Re}_{P}}\left(1+0.5 \operatorname{Re}_{P}^{0.687}\right), & \text { if } 1 \leq \operatorname{Re}_{P} \leq 1000 \\
0.44, & \text { if } \operatorname{Re}_{P}>1000 \\
\text { where } & \operatorname{Re}_{\mathrm{p}}=\frac{2 \mathrm{a}\left|\overrightarrow{\mathrm{u}}_{\mathrm{f}}-\overrightarrow{\mathrm{u}}_{\mathrm{p}}\right| \rho_{f}}{\mu_{f}}
\end{array}\right.
$$

Where $\vec{\mu}_{f}$ is the fluid dynamic viscosity. The modified version of the TAB breakup is based on the breakup regime and proportional relation determined the product droplets rate. PilchErdman approach modeled the droplet in high flow velocity. ReitzDiwakar model create a distinction among the stripping and bag breakup regimes [24].

\section{NUMERICAL IMPLEMENTATION SETUP}

Figure 1 schematically explains the procedure of the atomization and electrostatic spray painting process in the ERBS. In this process the paint droplet that distributed with high-velocity from the edge of the bell cup affected by the ionized airflow and shaping airflow which is injected from the nozzles and then impinging to the target surface.

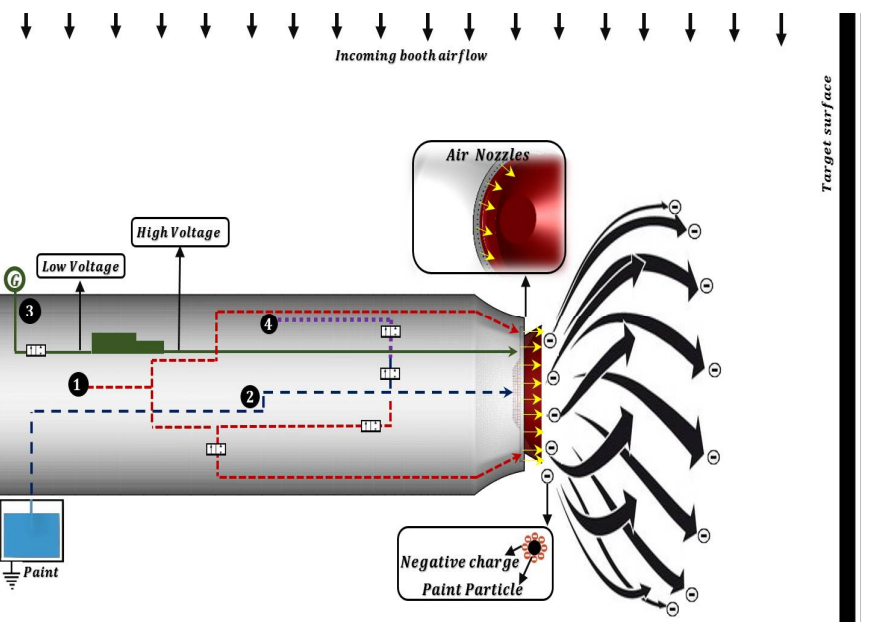

Figure 1. A description for the electrostatic spray coating procedure in the ERBS.

Figure 2 represents that structure quadrilateral mesh was used for almost entire computational domain. After examination of various grid size [9], we performed our simulation by using around 8.5 million cell. The frames in figure 2 illustrates that there is a very dense mesh near the cup of the ERBS, shaping air nozzles and target surface to capture the better the high turbulence flow. Figure 3 indicates the boundary conditions and computational domain which is consists of a circular cylinder of $2.6 \mathrm{~m} \times 2.8 \mathrm{~m}$ in diameter and length, respectively. 
The target distance is $0.2 \mathrm{~m}$ from the rotary bell surface. The airflow injected from the 90 holes with a diameter of $0.5 \mathrm{~mm}$.

\section{Results \& Discussion}

For a validation purpose, the dimensionless radial velocity values of the current study compared with the experimental data [6] in figure 4. These values extracted from the area very close to the rim of the bell cup surface where the turbulent layer starts to develop. Figure 5 showed the formation of the paint film on the surface of the bell cup. As this figure indicates, the current numerical result has the ability of capturing the paint particle distribution exactly same as the experimental result of Shen et al. [22]. The frames in figure 6 compare the subtended angle of the shaping air in two different rotational speeds of our study and the experimental snapshot of Darwish Ahmad et al. [11]. The angle of shaping air increased from $102^{\circ}$ to $131^{\circ}$ at the rotational speed of 20 and $50 \mathrm{kRPM}$, respectively, which is in good agreement with the experimental ones.

(a)

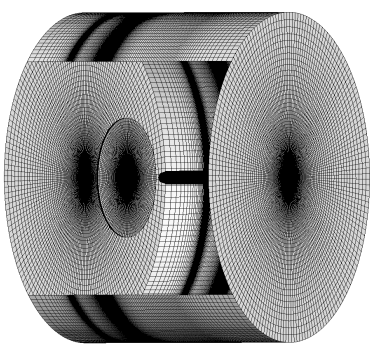

(c)

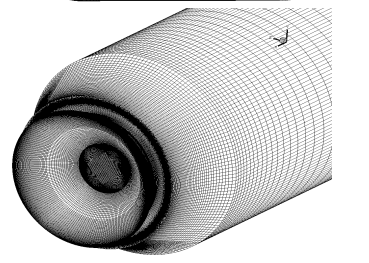

(b)

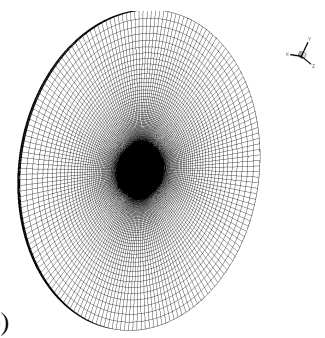

(d)

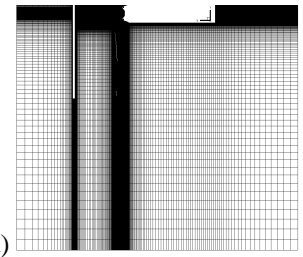

Figure 2. The computational mesh around the ERBS (a), figures show close-up view of (b) Target Surface (c) ERBS (d) 2D Views (Z-axis).

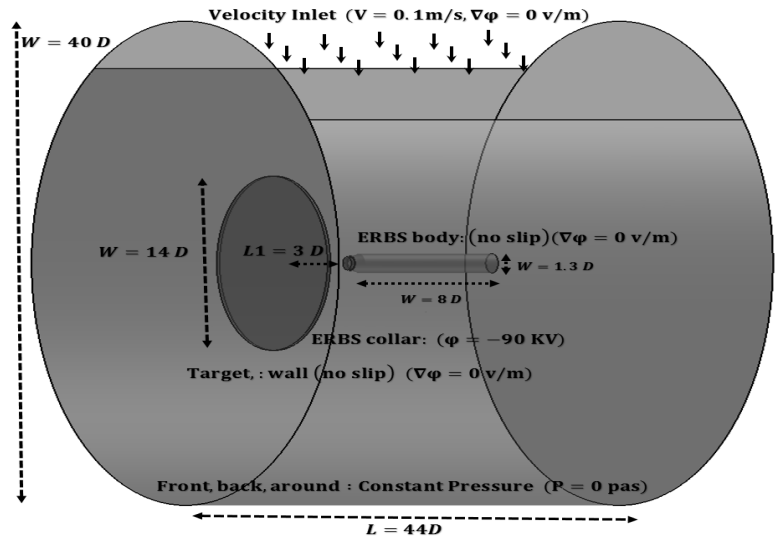

Figure 3. The dimensions of the computational domain with the boundary conditions and the electrostatic field.

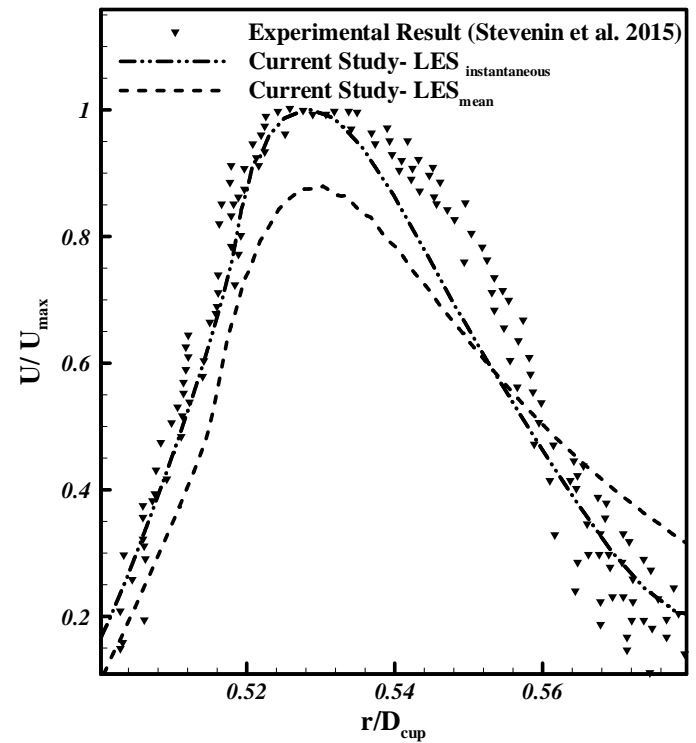

Figure 4. The dimensions of the computational domain with the boundary conditions and the electrostatic field.
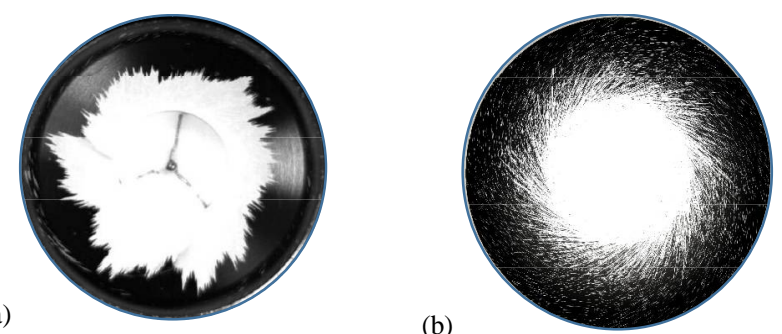

Figure 5. Paint film Propagation on the cup surface: (a) Experimental results of Shen et al. [22] (b) current study ( $\left.\omega_{\text {Bell }}=30 \mathrm{kRPM},{ }_{m_{\text {paint }}}=400 \mathrm{ml} / \mathrm{min}\right)$.

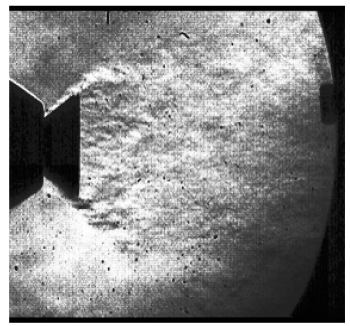

Exp.: $\alpha=136^{\circ}$

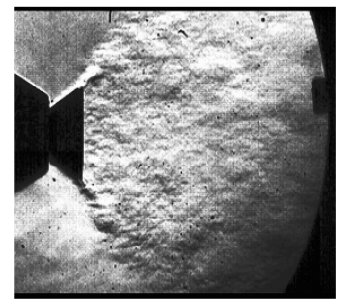

Exp.: $\alpha=96^{\circ}$
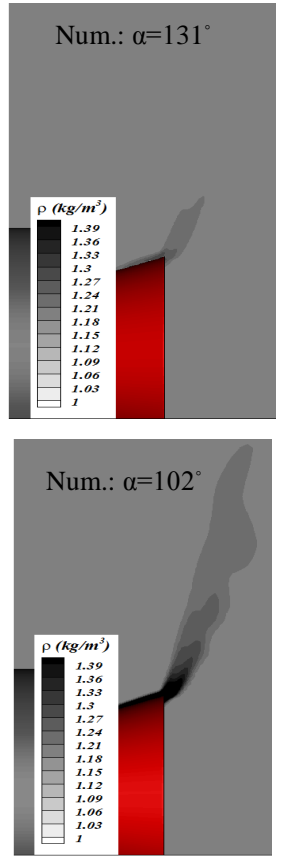

Figure 6. Shaping airflow outward of the rotary bell: Experimental results of Darwish Ahmad et al. [11] and contour of the density of the current numerical study. $\left(m_{\text {air }}=150\right.$ LPM , Reynolds $\left.=5.1 \times 10^{3}\right)$. 
Figure 7 shows six 3D temporal consecutive frames of sprayed paint droplet distribution for a complete cycle from the sprayer to the target that implemented with modified $\mathrm{TAB}$ [23] breakup approach and LES turbulence model. The droplet velocity near the bell cup surface is more than the cup tangential velocity $(150 \mathrm{~m} / \mathrm{s})$ and decrease significantly due to airflows interaction and drag force in the region far from the sprayer. The vortical overspray pattern growth and spread more as time goes.

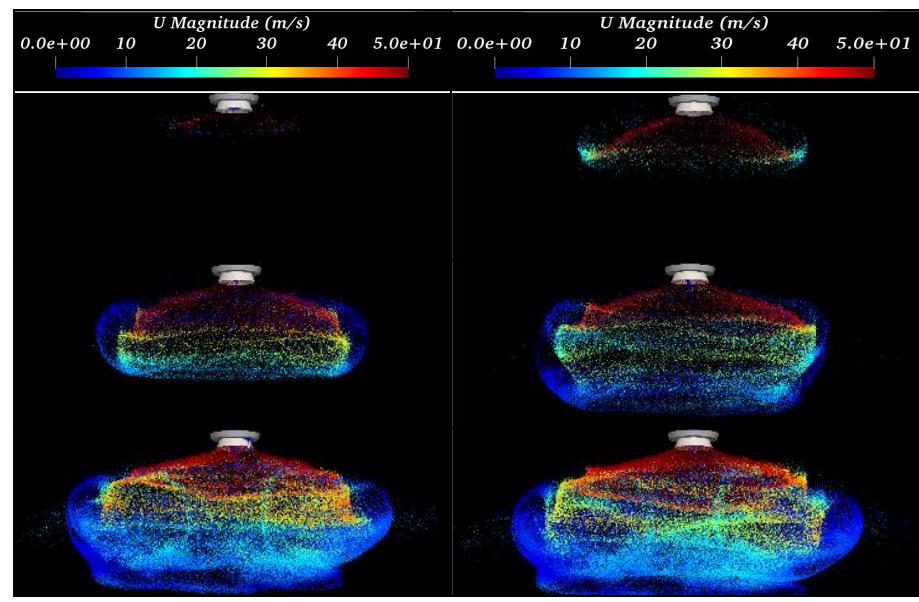

Figure 7. Spray pattern periodical time evolution, $t=5-55 \mathrm{~ms}$ $(\Delta t=10 \mathrm{~ms})\left(\right.$ modified TAB $\left.\omega_{\text {Bell }}=30 \mathrm{kRPM}\right)$.

Figure 8 illustrates the airflow pattern with streamline around ERBS that can be divided into outside and inside toroid-shape air vortices, blown airflow and trapped air vortices. As visualized in the frames the spray flow fully characterized, enlarges enough and reaches to self-similarity condition. TABLE I. compared the inner recirculation zone length $\left(L_{r z}\right)$, defined as the longitudinal length from the sprayer to the stagnation point, which is generated by the vortex breakdown mechanisms. By increasing the $\omega_{\text {Bell }}$, the values of $L_{r z}$ increases and almost reaches to the target.

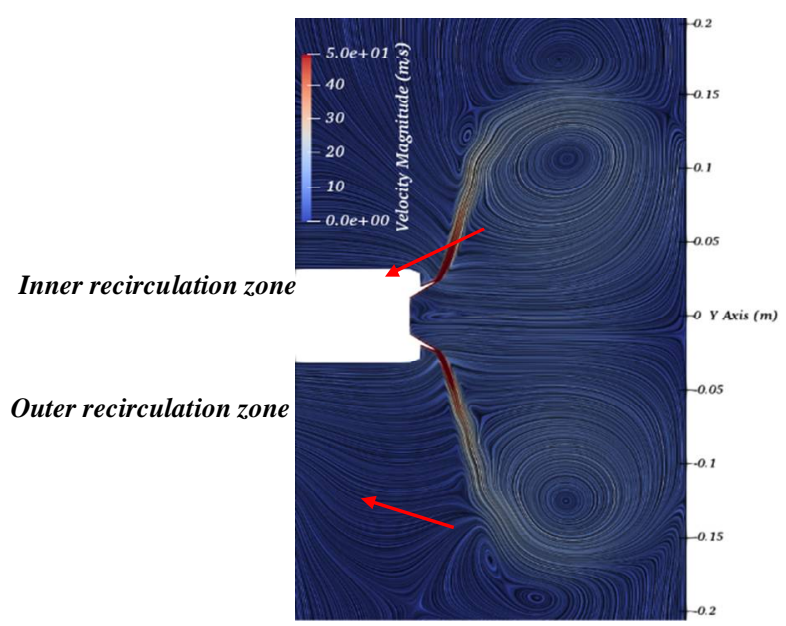

Figure 8 . Velocity contour $(\mathrm{m} / \mathrm{s})$ with streamline which is displayed by the LIC (Line Integral Convolution) method.
TABLE II. compares the Sauter Mean Diameter (SMD) of the droplets in different operational conditions. By increasing the rotational speed $\left(\Delta \omega_{\text {Bell }}=15 k R P M\right)$ the SMD values is reduced significantly around $\approx 52 \%$ in each step in the modified TAB model. This table also compares various breakup model. PilchErdman approach predicts larger values of SMD compare to other models. The comparison among two different bell cup diameter of sprayer shows the diameter of droplet is reduced in bigger bell cup size. The comparison between the different bell cup voltage ranges is indicated that the SMD values increase by growing the voltage.

TABLE I. The Inner recirculation length $\left(L_{r z} / d_{c u p}\right)$ for various $\omega_{\text {Bell }}$.

\begin{tabular}{|c|c|}
\hline$\omega_{\text {Bell }}$ & $L_{r z} / d_{\text {cup }}$ \\
\hline $25 k R P M$ & 1.64 \\
\hline $40 k R P M$ & 1.40 \\
\hline $55 k R P M$ & 2.38 \\
\hline
\end{tabular}

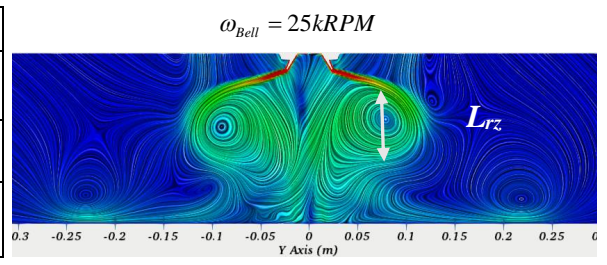

TABLE II. Sprayed droplet size distribution (SMD values).

\begin{tabular}{|c|c|c|}
\hline \multicolumn{3}{|c|}{ Modified TAB } \\
\hline (a) Rotational Speed & $S M D_{32}(\mu \mathrm{m})$ & $S M D_{10}(\mu \mathrm{m})$ \\
\hline$\omega_{\text {Bell }}=25 k R P M$ & 13.62 & 10.22 \\
\hline$\omega_{\text {Bell }}=40 k R P M$ & 7.42 & 6.12 \\
\hline$\omega_{\text {Bell }}=55 \mathrm{kRPM}$ & 3.84 & 3.11 \\
\hline (b) Bell Cup Diameter & $S M D_{32}(\mu \mathrm{m})$ & $S M D_{10}(\mu \mathrm{m})$ \\
\hline$D_{\text {cup }}=50 \mathrm{~mm}$ & 7.42 & 6.12 \\
\hline$D_{\text {cup }}=65 \mathrm{~mm}$ & 6.6 & 3.56 \\
\hline (c) Voltage Range & $S M D_{32}(\mu \mathrm{m})$ & $S M D_{10}(\mu \mathrm{m})$ \\
\hline Zero & 17.3 & 10.45 \\
\hline$-30 K V$ & 24.49 & 10.54 \\
\hline$-60 K V$ & 25.998 & 10.68 \\
\hline$-90 \mathrm{KV}$ & 27.49 & 10.69 \\
\hline \multicolumn{3}{|c|}{ PilchErdman } \\
\hline (d) Rotational Speed & $S M D_{32}(\mu \mathrm{m})$ & $S M D_{10}(\mu \mathrm{m})$ \\
\hline$\omega_{\text {Bell }}=25 \mathrm{kRPM}$ & 15.92 & 13.96 \\
\hline$\omega_{\text {Bell }}=40 \mathrm{kRPM}$ & 7.99 & 7.323 \\
\hline$\omega_{\text {Bell }}=55 \mathrm{kRPM}$ & 4.85 & 4.41 \\
\hline \multicolumn{3}{|c|}{ ReitzDiwakar } \\
\hline (e) Rotational Speed & $S M D_{32}(\mu \mathrm{m})$ & $S M D_{10}(\mu \mathrm{m})$ \\
\hline$\omega_{\text {Bell }}=25 k R P M$ & 12.99 & 11.365 \\
\hline$\omega_{\text {Bell }}=40 \mathrm{kRPM}$ & 5.87 & 5.368 \\
\hline$\omega_{\text {Bell }}=55 \mathrm{kRPM}$ & 3.2 & 3.0 \\
\hline
\end{tabular}

Figure 9 shows the constructed film pattern, thickness and uniformity for the case with $\omega_{\text {Bell }}=55 \mathrm{kRPM}$. The circular pattern of deposition, including non-homogenous and nonasymmetric, is indicated in this figure and this film diminishes sharply outside the circle. Figure 10 plotted the distribution of the paint flux in the target's radial direction to consider the effect of the various breakup model on filmbuilding. The film distribution for the different breakup models is almost 
identical. All models produced a specific peak at roughly $r \approx 2.0 R_{\text {Bell }}$ and no droplet landing beyond the mentioned area. The values of the film thickness decrease in order for the Reitz-Diwakar, modified TAB and the Pilch-Erdman, respectively, under the same operating conditions.

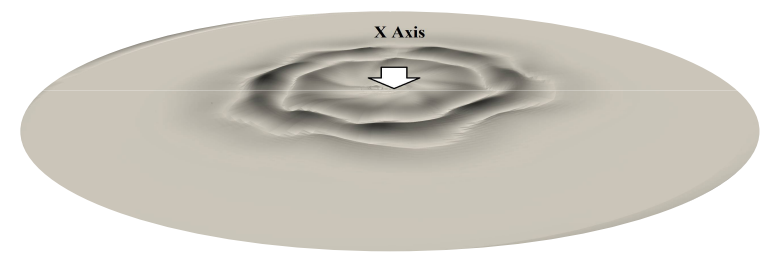

Figure 9. 3D paint film visualization on the target surface $\left(\omega_{\text {Bell }}=55 k R P M\right.$, scale factor $\left.S F_{\text {Film }}=1000\right)$.

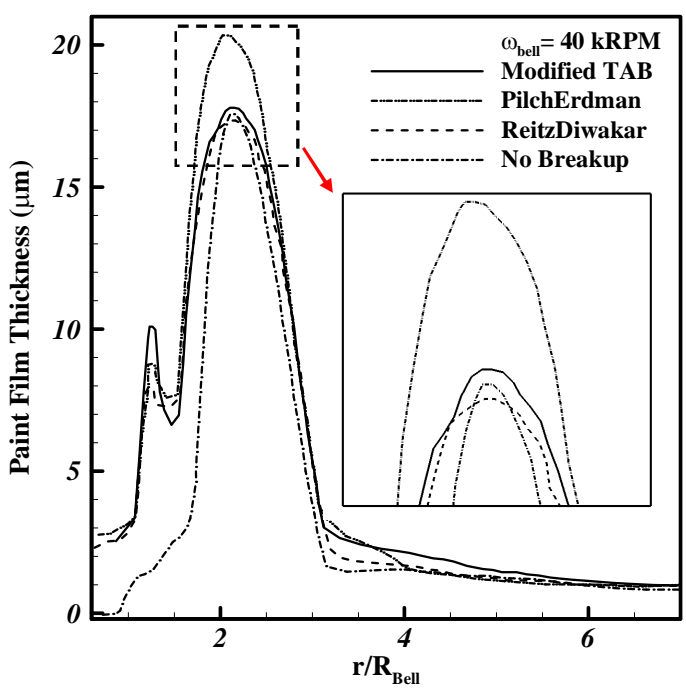

Figure 10. Distribution of paint film thickness for the various breakup models $\left(\omega_{\text {Bell }}=40 \mathrm{kRPM}\right)$.

Figure 11 indicates a droplet pattern instantaneous snapshot in two different bell cup voltage. The rebounded paint particles from the target surface constructed a bigger vortical torus shape in the lower voltage. In the higher voltage the spray cone shape is more uniform due to higher velocity of droplet and the coating transfer efficiency increase significantly.

(a) $0 \mathrm{kV}$

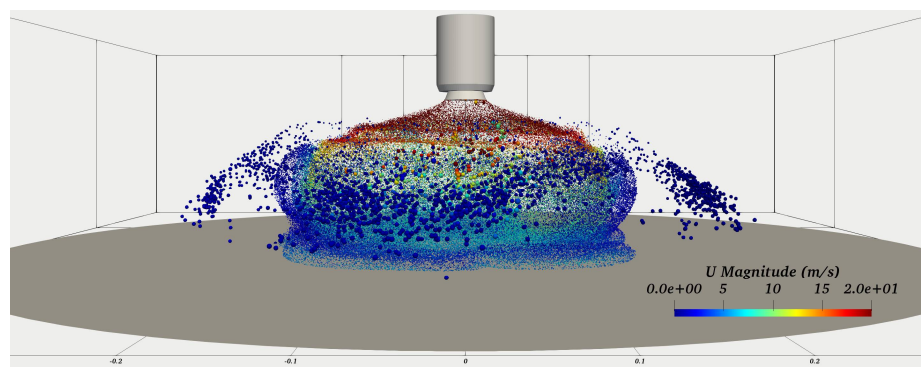

(b) $-20 \mathrm{kV}$

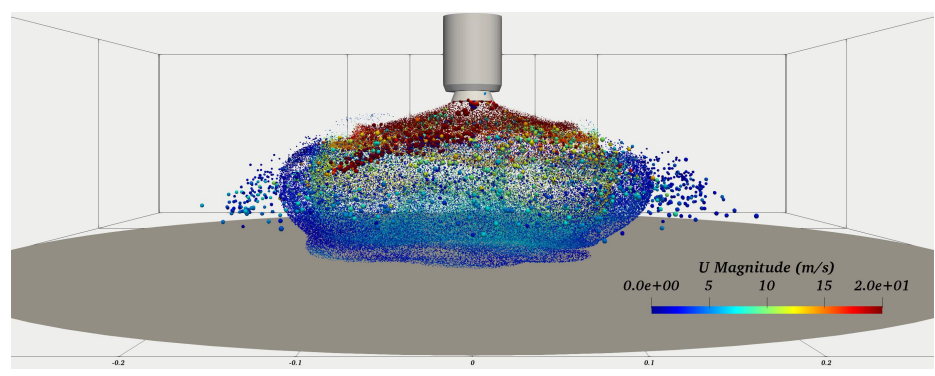

Figure 11. 3D views of the spray pattern for two different bell cup voltage and low value of charge to mass ratio $(q / m=0.5 \mathrm{mc} / \mathrm{kg})$.

\section{CONCLUSIONS}

The main contribution of the current work is to find a strong tool to simulate the spray transfer process in the ERBS by using the framework of the OpenFOAM. This work introduces a thorough understanding of the droplet trajectory and complex airflows by using various breakup models. Also, the size, mass, velocity, charge-to-mass ratio and disintegration of the injected droplet during the spraying was studied precisely. Also, the film formation on the target surface and transfer efficiency reported in different operational conditions.

\section{ACKNOWLEDGMENT}

This work was supported by Project “ INDTECH 4.0 -Novas Tecnologias para Fabricacao Inteligente” , project grant No. POCI-01- 0247-FEDER-026653. The research was also partly supported by CMAST Center for Mechanical and Aerospace Science and Technology, research unit $\mathrm{n}^{\circ} 151$ from Fundacao para a Ciencia e Tecnologia (Portugal).

\section{REFERENCES}

[1] Q. Ye, B. Shen, O. Tiedje, T. Bauernhansl, , J. Domnick, "Numerical and Experimental Study of Spray Coating Using Air-Assisted High Pressure Atomizers." At. Sprays, 25 (8) 643-656, 2015.

[2] N. Toljic, K. Adamiak, G.S.P. Castle, H.H. Kuo, , H.T. Fan, "3D Numerical Model of the Electrostatic Coating Process With Moving Objects Using a Moving Mesh.'” J. Electrostat., 70 499-504, 2012.

[3] V. Viti and J. Kulkarni, "CFD analysis of the electrostatic spray painting process with a rotating bell cup," In: ILASS Americas, 21st Annual Conference on Liquid Atomization and Spray Systems, Orlando, Florida, USA, 2008.

[4] P.J. O'Rourke and A.A. Amsden, "A spray/wall interaction submodel for the KIVA-3 wall film model," SAE transactions, pp.281-298, 2000.

[5] A. Darwish Ahmad, B.B. Singh, M. Doerre, A.M. Abubaker, M. Arabghahestani, A.A. Salaimeh and N.K. Akafuah, "Spatial Positioning and Operating Parameters of a Rotary Bell Sprayer: 3D Mapping of Droplet Size Distributions," Fluids, 4(3), p.165, 2019.

[6] C. Stevenin, Y. Bereaux, J.Y. Charmeau, , J. Balcaen , "Shaping air flow characteristics of a high-speed rotary-bell sprayer for automotive painting processes, ”J. Fluids Eng, 137 (11), 111304, 2015. 
[7] F. Rodrigues, A. Mushyam, J. Pascoa, M. Trancossi, "A new plasma actuator configuration for improved efficiency: The stair-shaped dielectric barrier discharge actuator." Journal of Physics D: Applied Physics, Vol. 52, 2019.

[8] A. Mushyam, F. Rodrigues, J.C. Pascoa, "A plasma-fluid model for EHD flow in DBD actuators and experimental validation," International Journal for Numerical Methods in Fluids, vol. 90, 2019.

[9] M.R. Pendar and J.C. Páscoa, "Numerical modeling of electrostatic spray painting transfer processes in rotary bell cup for automotive painting," International Journal of Heat and Fluid Flow, 80, p.108499, 2019.

[10] M.R. Pendar and J. Pascoa, "Numerical Investigation of Electrostatic Spray Painting Transfer Processes for Vehicle Coating," (No. 2019-011856). SAE Technical Paper, 2019.

[11] A. Darwish Ahmad, A. Abubaker, A. Salaimeh, and N. Akafuah, "Schlieren Visualization of Shaping Air during Operation of an Electrostatic Rotary Bell Sprayer: Impact of Shaping Air on Droplet Atomization and Transport," Coatings, 8(8), p.279, 2018.

[12] J. Wilson, S. Grib, A. Darwish Ahmad, M. Renfro, S. Adams and A. Salaimeh, "Study of near-cup droplet breakup of an automotive electrostatic rotary bell (esrb) atomizer using high-speed shadowgraph imaging," Coatings, 8(5), p.174, 2018.

[13] H. Fujimoto, H.O.R.I. Tsukasa and J. Senda, "Effect of breakup model on diesel spray structure simulated by large eddy simulation," (No. 2009-24-0024). SAE Technical Paper, 2009.

[14] M. H. Rami and J. Pascoa, "Numerical analysis of a cycloidal rotor under diverse operating conditions and altitudes," In SAE Technical Paper Series, SAE International, 2019.

[15] M. Habibnia and J. Pascoa, "ANN assisted flow modeling and analysis for a cyclorotor in ground effect". Aerospace Science and Technology, 95, dec, p. 105495, 2019.

[16] M. H. Rami, S. Vakilipour, M. H. Sabour, R. Riazi and H. Hassannia, "Shear layer investigation through a high-load cascade in low-pressure gas turbine conditions," International Journal of Mechanical and Mechatronics Engineering, 2019.

[17] S. Vakilipour, M. Habibnia, M. H. Sabour, R. Riazi and M. Mohammadi, "Surface pressure characteristics of a highly loaded turbine blade at design and off-design conditions a CFD methodology," Thermophysics and Aeromechanics, 24(3), may, pp. 469-482, 2017.

[18] M.R. Pendar, E. Roohi, "Cavitation characteristics around a sphere: an LES investigation,” Int. J. Multiphase Flow. 98, pp. 1-23, 2018.

[19] A. Kolahan, E. Roohi and M.R.Pendar, "Wavelet analysis and frequency spectrum of cloud cavitation around a sphere," Ocean Engineering, 182, pp.235-247, 2019.

[20] E. Roohi, M.R. Pendar and A. Rahimi, "Simulation of three-dimensional cavitation behind a disk using various turbulence and mass transfer models," Applied Mathematical Modelling, 40(1), pp.542-564, 2016.

[21] M.R. Pendar and E. Roohi, "Detailed investigation of cavitation and supercavitation around different geometries using various turbulence and mass transfer models," In Journal of Physics: Conference Series (Vol. 656, No. 1, p. 012070). IOP Publishing, 2015.

[22] B. Shen, Q. Ye, N. Guettler, O. Tiedje, and J. Domnick, "Primary breakup of a non-Newtonian liquid using a high-speed rotary bell atomizer for spray-painting processes, " Journal of Coatings Technology and Research, pp.1-16, 2019.

[23] F.X. Tanner, "Liquid jet atomization and droplet breakup modeling of non-evaporating diesel fuel sprays, " SAE transactions, pp.127-140, 1997.

[24] P. Ghadimi, M. Yousefifard, and H. Nowruzi, "Applying Different Strategies within OpenFOAM to Investigate the Effects of Breakup and Collision Model on the Spray and in-Cylinder Gas Mixture Attribute," Journal of Applied Fluid Mechanics, 9(6), 2016. 